\title{
Novel $B$-decay signatures of light scalars at high energy facilities
}

\author{
Andrew Blance, ${ }^{1,2}$ Mikael Chala, ${ }^{1,3}$ Maria Ramos $\odot,{ }^{4}$ and Michael Spannowsky ${ }^{1}$ \\ ${ }^{1}$ Institute of Particle Physics Phenomenology, Physics Department, Durham University, \\ Durham DH1 3LE, United Kingdom \\ ${ }^{2}$ Institute for Data Science, Durham University, Durham DH1 3LE, United Kingdom \\ ${ }^{3}$ CAFPE and Departamento de Física Teórica y del Cosmos, Universidad de Granada, \\ E18071 Granada, Spain \\ ${ }^{4}$ Laboratório de Instrumentação e Física Experimental de Partículas, Departamento de Física da \\ Universidade do Minho, Campus de Gualtar, 4710-057 Braga, Portugal
}

(Received 6 August 2019; published 6 December 2019)

\begin{abstract}
We study the phenomenology of light scalars of masses $m_{1}$ and $m_{2}$ coupling to heavy flavor-violating vector bosons of mass $m_{V}$. For $m_{1,2} \lesssim$ few $\mathrm{GeV}$, this scenario triggers the rare $B$ meson decays $B_{s}^{0} \rightarrow 3 \mu^{+} 3 \mu^{-}, B^{0} \rightarrow 3 \mu^{+} 3 \mu^{-}, B^{+} \rightarrow K^{+} 3 \mu^{+} 3 \mu^{-}$, and $B_{s}^{0} \rightarrow K^{0 *} 3 \mu^{+} 3 \mu^{-}$; the last two being the most important ones for $m_{1} \sim m_{2}$. None of these signals have been studied experimentally; therefore, we propose analyses to test these channels at the LHCb. We demonstrate that the reach of this facility extends to branching ratios as small as $6.0 \times 10^{-9}, 1.6 \times 10^{-9}, 5.9 \times 10^{-9}$, and $1.8 \times 10^{-8}$ for the aforementioned channels, respectively. For $m_{1,2} \gg \mathcal{O}(1) \mathrm{GeV}$, we show that slightly modified versions of current multilepton and multitau searches at the LHC can probe wide regions of the parameter space of this scenario. Altogether, the potential of the searches we propose outperform other constraints such as those from meson mixing.
\end{abstract}

DOI: $10.1103 /$ PhysRevD.100.115015

\section{INTRODUCTION}

Searches for new physics in final states often considered as "standard candles," most notably in searches for supersymmetry (SUSY), have not provided any evidence of physics beyond the Standard Model (BSM) so far. This fact does not necessarily disproves low energy SUSY or other popular BSM extensions [1], such as composite Higgs models (CHM) [2,3]. However, it supports the search for new physics in radically new and still unexplored channels.

In this paper, we focus on light singlet scalars $a_{1,2}$ that can be produced in rare decays of $B$ mesons mediated by heavy flavor-violating vector bosons $V$. This scenario is especially motivated, as it arises naturally in nonminimal CHMs [4-9]. ( $V$ and $a_{1,2}$ can be seen as the counterparts of the $\rho$ and the pions in QCD.) Likewise, such vector boson can explain the apparent anomalies observed in tests of lepton flavor universality [10-17]. Moreover, the bounds on such vector boson are weakened when it decays into lighter composite resonances [17], such as the

Published by the American Physical Society under the terms of the Creative Commons Attribution 4.0 International license. Further distribution of this work must maintain attribution to the author(s) and the published article's title, journal citation, and DOI. Funded by SCOAP ${ }^{3}$. aforementioned scalars. Finally, also supersymmetric models can trigger similar decays, mediated by scalar and pseudoscalar sgoldstino particles [18].

If, similarly to the Higgs boson, the scalars couple stronger to the muon than to the electron, processes such as $B_{s}^{0} \rightarrow a_{1} a_{2}$ can lead to four muon final states. To the best of our knowledge, the corresponding signal has been studied experimentally only at the LHCb [19], the most stringent limit being $\mathcal{B}\left(B_{s}^{0} \rightarrow 2 \mu^{+} 2 \mu^{-}\right)<2.5 \times 10^{-9}$.

However, there are different reasons to consider alternative $B$ meson decay modes. To start with, the partial width for $a_{2} \rightarrow a_{1} a_{1}$ can very easily dominate over the corresponding leptonic width. In this case, six-muon final states rather than four muon ones are to be studied. And second, the scalars couple to the mediator as a vector current $\sim a_{1} \partial a_{2}$. When the latter is conserved, namely for $m_{1} \sim$ $m_{2}$ (and in particular in the massless limit), the $B$ meson decay into such scalars vanishes. In other words, $\Gamma\left(B_{s}^{0} \rightarrow a_{1} a_{2}\right) \sim\left(m_{1}^{2}-m_{2}^{2}\right) / m_{B}$. In this regime, one should rather explore three body decays of $B$ with emitted mesons. In this work, we focus mostly on $B^{+} \rightarrow K^{+} 3 \mu^{+} 3 \mu^{-}$. (The inclusion of conjugate modes of charged decays is implied throughout the paper.)

We also extend previous works on this topic $[18,20,21]$ by studying the regime of large scalar masses. In such regime, $a_{1,2}$ can no longer show up in rare decays of $B$ mesons. However, they can appear in decays of the vector 
mediator if it is at the $\mathrm{TeV}$ scale and therefore be produced in $p p$ collisions at the LHC.

This article is organized as follows. In Sec. II, we provide the Lagrangian relevant for our study and define the region of the parameter space of phenomenological interest. In Sec. III, we focus on the regime $m_{1,2} \lesssim$ few Gev and provide analyses for the LHCb and estimate the reach for different $B$ decays. We do not circumscribe to any particular value of $m_{1,2}$, but rather scan over different values of these. In Sec. IV, we focus instead on the regime $m_{1,2}>$ few $\mathrm{GeV}$ and study the corresponding LHC signatures.

Unless otherwise stated, all limits given in this article stand for $95 \%$ CL.

We conclude in Sec. V, while we dedicate Appendix to building a complete model that predicts definite values of several of the parameters that we scan over.

\section{FRAMEWORK}

Let us consider the Lagrangian of the Standard Model (SM) extended with a heavy vector $V$ and two light scalars $a_{1}, a_{2}$. The relevant Lagrangian before electroweak symmetry breaking (EWSB) (on the basis in which up quark and lepton Yukawas are diagonal) reads

$$
\begin{aligned}
L= & \frac{1}{2} m_{V}^{2} V_{\mu} V^{\mu}+\frac{1}{2} m_{1}^{2} a_{1}^{2}+\frac{1}{2} m_{2}^{2} a_{2}^{2}+m_{12} a_{2} a_{1}^{2}+\ldots \\
& +V^{\mu}\left[g_{12} a_{1} \overleftrightarrow{\partial_{\mu}} a_{2}+g_{q q}\left(\overline{q_{L}} \gamma_{\mu} q_{L}+\text { H.c. }\right)\right],
\end{aligned}
$$

with $m_{V} \gg m_{1,2}$. The ellipsis stands for terms not relevant for this study. Without loss of generality, we assume $m_{2}>m_{1}$. The scalars $a_{1,2}$ can be more naturally thought of as the real and imaginary components of a complex field $\Phi$; the Lagrangian being invariant under $\Phi \rightarrow \exp (i \theta) \Phi$ up to $\mathcal{O}\left(1-m_{2} / m_{1}, m_{12}\right)$. In Appendix, we match a concrete $\mathrm{CHM}$ to the Lagrangian above.

Assuming that $V$ interacts mostly with the third generation quarks, after EWSB it couples to $\overline{b_{L}} b_{L}$ and $\overline{t_{L}} t_{L}$ as well as $\overline{b_{L}} s_{L}+$ H.c. with strengths $\sim g_{q q}$ and

$$
g_{s b} \equiv g_{q q} V_{t s}^{\mathrm{CKM}} V_{t b}^{\mathrm{CKM}} \sim 0.04 g_{q q},
$$

respectively.

We distinguish two different regimes depending on the masses of the scalars: $1 \mathrm{GeV} \lesssim m_{1,2} \lesssim 4 \mathrm{GeV}$ (low-mass regime) and $m_{1,2}>4 \mathrm{GeV}$ (high-mass regime). Likewise, we consider two possible scenarios for the couplings of $a_{1,2}$ to the fermions. First, we assume that $a_{1,2}$ are muonphilic. As a second possibility, we assume that they couple only to the SM leptons and with Higgs-like strength, namely $\sim g_{1,2} y_{\ell} a_{1,2} \ell^{+} \ell^{-}$, with $y_{\ell}$ the SM Yukawa couplings and $g_{1,2}$ free dimensionless parameters and lepton independent.

In the low-mass regime, $a_{1}$ decays mostly into muons irrespectively of whether it is muonphilic or just leptophilic. In the high-mass regime, it decays mostly into taus unless it is muonphilic.

Regarding the decay of $a_{2}$, if $m_{2}>2 m_{1}$, then $a_{2}$ can either decay into $a_{1} a_{1}$ or into lepton pairs, depending on $m_{12} / g_{2}$,

$$
\begin{gathered}
\Gamma\left(a_{2} \rightarrow \ell^{+} \ell^{-}\right)=\frac{g_{2}^{2} y_{\ell}^{2}}{8 \pi}\left(1-\frac{4 m_{\ell}^{2}}{m_{2}^{2}}\right)^{3 / 2} m_{2}, \\
\Gamma\left(a_{2} \rightarrow a_{1} a_{1}\right)=\frac{m_{12}^{2}}{8 \pi m_{2}}\left(1-\frac{4 m_{1}^{2}}{m_{2}^{2}}\right)^{1 / 2} .
\end{gathered}
$$

In what follows, we assume that $m_{12} / m_{2} \gg g_{2} y_{\ell}$ in this regime, so that $\mathcal{B}\left(a_{2} \rightarrow a_{1} a_{1}\right) \gg \mathcal{B}\left(a_{2} \rightarrow \ell^{+} \ell^{-}\right)$. Note that this inequality holds almost trivially, since one expects $m_{12} \sim m_{2}$ whereas the Yukawas are tiny.

If instead $m_{2}<2 m_{1}, a_{2}$ can either decay into pairs of leptons as before, or into $a_{1} \ell^{+} \ell^{-}$with width

$\Gamma\left(a_{2} \rightarrow a_{1} \ell^{+} \ell^{-}\right) \sim \frac{\left(g_{1} y_{\ell}\right)^{2}}{64 \pi^{3} m_{2}^{3}} m_{12}^{2} m_{1}^{2}\left(1+\frac{m_{2}}{m_{1}}\right)\left(\frac{m_{2}}{m_{1}}-1\right)^{5}$.

This decay mode dominates if $g_{1} \gtrsim 100 g_{2}$. We assume this hierarchy hereafter. Thus, for example, for $g_{1}=3$ and $g_{2}=0.01, a_{2}$ decays always into four leptons mediated by $a_{1}$, which can be either on shell or off shell. Also, they both have widths smaller than $10 \mathrm{MeV}$ and lifetime shorter than 10 fs. As a consequence, both $a_{1,2}$ would seem to have vanishing experimentally measurable widths and flight distances. Furthermore, note that the Yukawa suppression helps also avoiding bounds from $B A B A R$ and even the future Belle-II [22].

At low energies, the vector boson $V$ triggers $B$ meson decays into the light scalars; see Fig. 1. Depending on the relative size between $m_{B}$ and $m_{1,2}$, we distinguish the following two cases:

(i) If $m_{B}>m_{1}+m_{2}$, we have $B_{s}^{0} \rightarrow a_{1} a_{2}$.

(ii) If $m_{B}<m_{1}+m_{2}$ and $m_{B}>3 m_{1}$, we have instead $B_{s}^{0} \rightarrow a_{1} a_{1} a_{1}$. (Other three body decays, e.g., $B_{s}^{0} \rightarrow a_{1} \mu^{+} \mu^{-}$are subdominant due to the Yukawa suppression.)

If $m_{B}>m_{1}+m_{2}+m_{K}$, we also have $B^{+} \rightarrow K^{+} a_{1} a_{2}$. We do not consider any other cases in this paper; see Fig. 2.
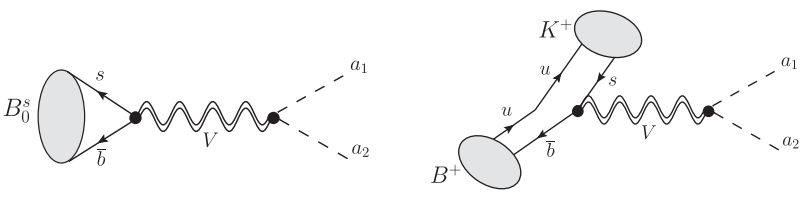

FIG. 1. Tree level Feynman diagram for the decays $B_{s}^{0} \rightarrow a_{1} a_{2}$ (left) and $B^{+} \rightarrow K^{+} a_{1} a_{2}$ (right). 


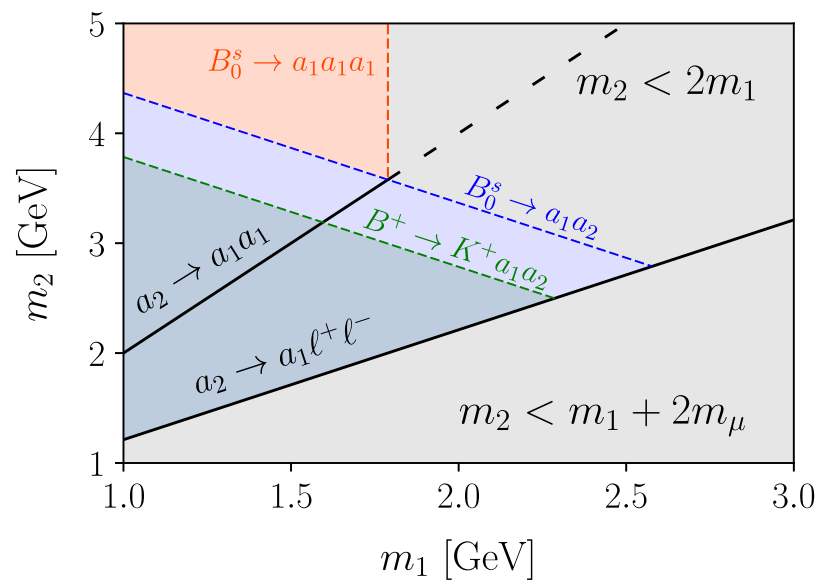

FIG. 2. Dominant decays taking place in the different regions of the plane $\left(m_{1}, m_{2}\right)$. The gray areas are not considered in this analysis.

The decay width for $B_{s}^{0} \rightarrow a_{1} a_{2}$ reads

$$
\Gamma=\frac{f_{B}^{2}}{16 \pi m_{V}^{4}}\left(g_{s b} g_{12}\right)^{2} \frac{\left(m_{1}^{2}-m_{2}^{2}\right)^{2}}{m_{B}} \mathcal{K}\left(\frac{m_{1}}{m_{B}}, \frac{m_{2}}{m_{B}}\right),
$$

with

$$
\mathcal{K}(x, y)=\left[x^{4}+\left(1-y^{2}\right)^{2}-2 x^{2}\left(1+y^{2}\right)\right]^{1 / 2}
$$

and $f_{B} \sim 0.23 \mathrm{GeV}$ [23].

The amplitude for $B_{s}^{0} \rightarrow a_{1} a_{1} a_{1}$ reads

$$
\mathcal{M}=2 g_{s b} g_{12} \frac{f_{B} m_{12}}{m_{V}^{2}}\left[\frac{q_{12}^{2}-m_{1}^{2}}{q_{12}^{2}-m_{2}^{2}}+\frac{q_{23}^{2}-m_{1}^{2}}{q_{23}^{2}-m_{2}^{2}}+\frac{q_{13}^{2}-m_{1}^{2}}{q_{13}^{2}-m_{2}^{2}}\right],
$$

where we have defined the transferred momenta $q_{12}^{2}=$ $\left(p_{1}+p_{2}\right)^{2}, \quad q_{23}^{2}=\left(p_{2}+p_{3}\right)^{2}$ and $q_{13}^{2}=\left(p_{1}+p_{3}\right)^{2}=$ $3 m_{1}^{2}+m_{B}^{2}-q_{12}^{2}-q_{23}^{2}$. After integrating over $q_{23}^{2}$, we obtain

$$
\frac{d \Gamma}{d q_{12}^{2}}=\frac{\left(g_{s b} g_{12}\right)^{2} m_{2}^{2}}{384 \pi^{3} m_{B}^{3}}\left(\frac{f_{B} m_{12}}{m_{V}^{2}}\right)^{2} F\left[\frac{m_{1}}{m_{2}}, \frac{m_{B}}{m_{2}}, \frac{q_{12}}{m_{2}}, \frac{q_{23}}{m_{2}}\right]_{\left(q_{23}^{2}\right)^{\min }}^{\left(q_{23}^{2} \max \right.},
$$

with

$$
\begin{aligned}
F(x, y, w, v)= & \left(1-x^{2}\right)^{2}\left[\frac{1}{1-v^{2}}+\frac{1}{3 x^{2}+y^{2}-w^{2}-v^{2}-1}\right]+\frac{v^{2}\left(2+x^{2}-3 w^{2}\right)^{2}}{\left(w^{2}-1\right)^{2}}+2\left(x^{2}-1\right) \\
& \times\left\{\frac{3 x^{4}+x^{2}\left(3+y^{2}-9 w^{2}\right)+y^{2}\left(2-3 w^{2}\right)+3\left(w^{4}+w^{2}-1\right)}{\left(w^{2}-1\right)\left(3 x^{2}+y^{2}-w^{2}-2\right)}\right. \\
& \left.\times\left[\log \left(v^{2}-1\right)-\log \left(1+w^{2}+v^{2}-3 x^{2}-y^{2}\right)\right]\right\},
\end{aligned}
$$

which should be evaluated at

$$
\begin{aligned}
& \left(q_{23}^{2}\right)^{\max }=\left(E_{2}^{*}+E_{3}^{*}\right)^{2}-\left(\sqrt{E_{2}^{* 2}-m_{1}^{2}}-\sqrt{E_{3}^{* 2}-m_{1}^{2}}\right)^{2} \\
& \left(q_{23}^{2}\right)^{\min }=\left(E_{2}^{*}+E_{3}^{*}\right)^{2}-\left(\sqrt{E_{2}^{* 2}-m_{1}^{2}}+\sqrt{E_{3}^{* 2}-m_{1}^{2}}\right)^{2},
\end{aligned}
$$

where $E_{2}^{*} \equiv q_{12} / 2$ and $E_{3}^{*} \equiv\left(m_{B}^{2}-q_{12}^{2}-m_{1}^{2}\right) /\left(2 q_{12}\right)$. The final width is obtained integrating over $q_{12}^{2}$ between $4 m_{1}^{2}$ and $\left(m_{B}-m_{1}\right)^{2}$.

In the limit $m_{1}, m_{2} \rightarrow 0$, the integrated width simplifies to

$$
\Gamma \sim \frac{3\left(g_{s b} g_{12}\right)^{2}}{256 \pi^{3}} \frac{f_{B}^{2} m_{12}^{2}}{m_{V}^{4}} m_{B}
$$

Finally, the amplitude for $B^{+} \rightarrow K^{+} a_{1} a_{2}$ is given by

$$
\mathcal{M}=-\frac{g_{s b} g_{12}}{m_{V}^{2}}\left\langle K\left(p_{3}\right)\left|\bar{s} \gamma_{\mu} b\right| B(p)\right\rangle\left(p_{2}-p_{1}\right)^{\mu},
$$

with

$$
\begin{aligned}
\left\langle K\left(p_{3}\right)\left|\bar{s} \gamma_{\mu} b\right| B(p)\right\rangle= & f_{+}\left(q^{2}\right)\left[\left(p+p_{3}\right)_{\mu}-\frac{m_{B}^{2}-m_{K}^{2}}{q^{2}} q_{\mu}\right] \\
& +f_{0}\left(q^{2}\right) \frac{m_{B}^{2}-m_{K}^{2}}{q^{2}} q_{\mu}
\end{aligned}
$$

and again $q^{2}=\left(p-p_{3}\right)^{2}$ is the transferred momentum, ranging from $\left(m_{1}+m_{2}\right)^{2}<q^{2}<\left(m_{B}-m_{K}\right)^{2}$. The contraction of this matrix element with $\left(p_{2}-p_{1}\right)$ in Eq. (13)) simplifies to

$$
\begin{aligned}
\mathcal{M}= & -\frac{g_{s b} g_{12}}{m_{V}^{2}}\left\{\frac{\left(m_{B}^{2}-m_{K}^{2}\right)\left(m_{2}^{2}-m_{1}^{2}\right)}{q^{2}}\left[f_{0}\left(q^{2}\right)-f_{+}\left(q^{2}\right)\right]\right. \\
& \left.+\left[2\left(p_{2}+p_{3}\right)^{2}+q^{2}-m_{1}^{2}-m_{2}^{2}-m_{B}^{2}-m_{K}^{2}\right] f_{+}\left(q^{2}\right)\right\} .
\end{aligned}
$$

For convenience, we trade these variables for $M_{12}^{2} \equiv m_{2}^{2}-$ $m_{1}^{2}$ and $M_{B K}^{2} \equiv m_{B}^{2}-m_{K}^{2}$, getting 


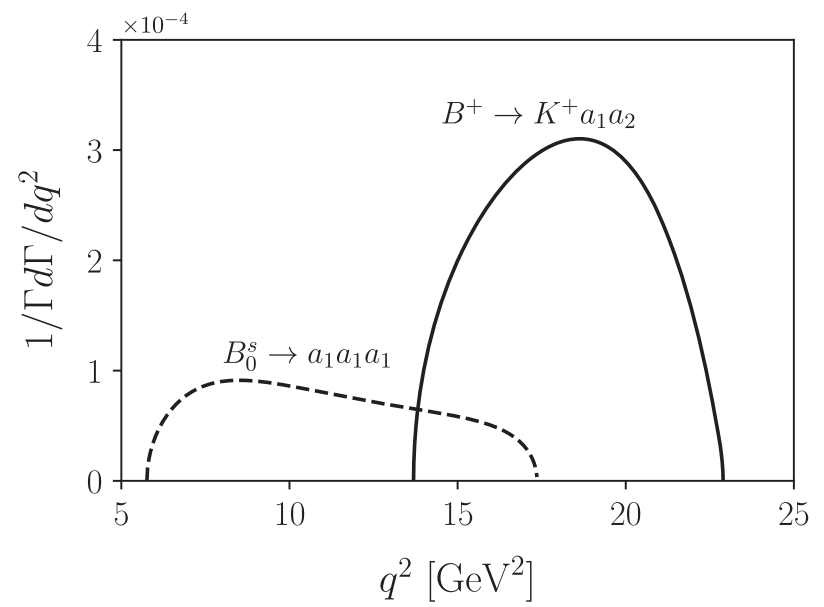

FIG. 3. Differential branching ratios as a function of $q^{2}=$ $\left(p_{1}+p_{2}\right)^{2}$. We have fixed $m_{1}=1.2 \mathrm{GeV}, g_{s b}=g_{12}=1$, $m_{V}=1 \mathrm{TeV}$, and $m_{12}=5 \mathrm{GeV}$. Due to the different kinematic regions where these decays take place, we have set $m_{2}=2.5 \mathrm{GeV}$ and $m_{2}=5 \mathrm{GeV}$ for the $B^{+} \rightarrow K^{+} a_{1} a_{2}$ and $B_{0}^{s} \rightarrow a_{1} a_{1} a_{1}$, respectively.

$$
\frac{d \Gamma}{d q^{2}}=\frac{\left(g_{s b} g_{12}\right)^{2}}{768 \pi^{3} m_{V}^{4} m_{B}^{3}} F\left(q^{2}\right),
$$

with

$$
\begin{aligned}
F\left(q^{2}\right)= & \frac{1}{q^{2}}\left[\frac{\left(M_{B K}^{2}+q^{2}\right)^{2}}{q^{4}}-4 \frac{m_{B}^{2}}{q^{2}}\right]^{1 / 2} \\
& \times\left[\frac{\left(M_{12}^{2}+q^{2}\right)^{2}}{q^{4}}-4 \frac{m_{2}^{2}}{q^{2}}\right]^{1 / 2}\left\{3 M_{B K}^{4} M_{12}^{4}\left|f_{0}\left(q^{2}\right)\right|^{2}\right. \\
& +\left[q^{4}+2 q^{2}\left(M_{B K}^{2}-2 m_{B}^{2}\right)+M_{B K}^{4}\right] \\
& \left.\times\left[q^{4}+2 q^{2}\left(M_{12}^{2}-2 m_{2}^{2}\right)+M_{12}^{4}\right]\left|f_{+}\left(q^{2}\right)\right|^{2}\right\} .
\end{aligned}
$$

Following Ref. [24], we parametrize the form factor as

$$
f_{+}\left(q^{2}\right)=\frac{r_{1}}{\left(1-q^{2} / m^{2}\right)}+\frac{r_{2}}{\left(1-q^{2} / m^{2}\right)^{2}},
$$

with $r_{1}=0.162, \quad r_{2}=0.173, \quad$ and $m^{2}=5.41^{2} \mathrm{GeV}^{2}$. Similarly,

$$
f_{0}\left(q^{2}\right)=\frac{r_{2}}{\left(1-q^{2} / m_{\mathrm{fit}}^{2}\right)}
$$

with $r_{2}=0.330$ and $m_{\text {fit }}^{2}=37.46 \mathrm{GeV}^{2}$. Finally, in the approximation $m_{1}, m_{2}, m_{K} \rightarrow 0$, and $f_{0}, f_{+}\left(q^{2}\right) \rightarrow 1$, we obtain

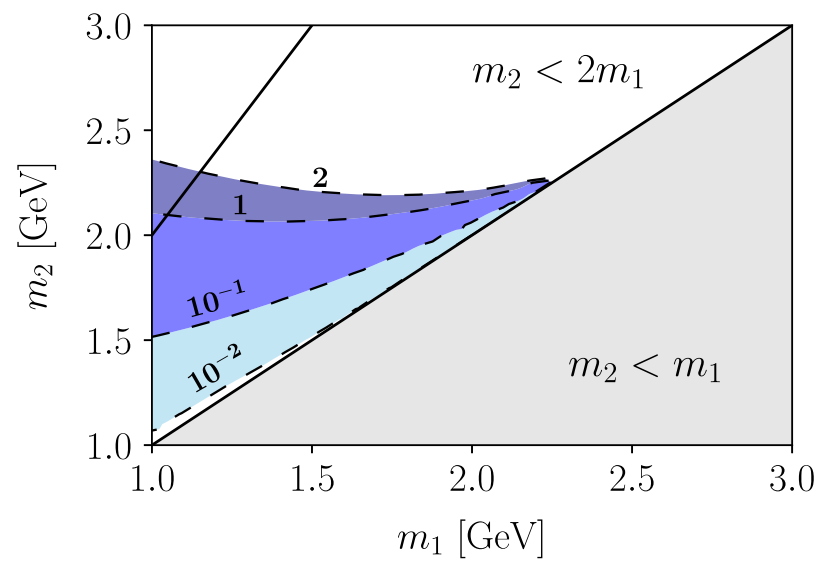

FIG. 4. Value of $\Gamma\left(B_{s}^{0} \rightarrow a_{1} a_{2}\right) / \Gamma\left(B^{+} \rightarrow K^{+} a_{1} a_{2}\right)$ in the plane $\left(m_{1}, m_{2}\right)$. This ratio vanishes along the line $m_{1}=m_{2}$.

$$
\Gamma \sim \frac{\left(g_{s b} g_{12}\right)^{2}}{3072 \pi^{3} m_{V}^{4}} m_{B}^{5}
$$

In Fig. 3, we show the magnitude of three body decays under consideration and their dependence with $\left(p_{1}+p_{2}\right)^{2}$. In Fig. 4, we show the ratio of $\Gamma\left(B_{s}^{0} \rightarrow a_{1} a_{2}\right)$ to $\Gamma\left(B^{+} \rightarrow K^{+} a_{1} a_{2}\right)$. It is very worth noting that it vanishes in the limit $m_{1} \rightarrow m_{2}$; see also Eq. (6). In this regime, searches for $B_{s}^{0}$ decaying only to muons are irrelevant; extra mesons have to be tagged instead. There are however no analyses (not even prospects) in this respect, and this is a gap that we try to overcome in this work.

At high energies, $V$ can be produced on shell in $p p$ collisions initiated by bottom quarks and subsequently decay into third generation quarks and into $a_{1} a_{2}$ with respective widths,

$$
\begin{gathered}
\Gamma(V \rightarrow q \bar{q})=\frac{g_{q q}^{2}}{8 \pi}\left(1-\frac{m_{q}^{2}}{m_{V}^{2}}\right)\left(1-\frac{4 m_{q}^{2}}{m_{V}^{2}}\right)^{1 / 2} m_{V}, \\
\Gamma\left(V \rightarrow a_{1} a_{2}\right)=\frac{g_{12}^{2}}{48 \pi}\left[1-2 \frac{m_{1}^{2}+m_{2}^{2}}{m_{V}^{2}}+\frac{\left(m_{2}^{2}-m_{1}^{2}\right)^{2}}{m_{V}^{4}}\right]^{3 / 2} m_{V},
\end{gathered}
$$

with $q=t, b$. Note that the scalar decay mode dominates already for $g_{12} \gtrsim 3 g_{q q}$.

\section{LOW-MASS REGIME AT THE LHCb}

In the low-mass regime, the smoking gun signature of the Lagrangian in Eq. (1) is rare decay of $B$ mesons into final states containing six muons (and possibly other lighter mesons). Let us focus first on the channel $B_{s}^{0} \rightarrow 3 \mu^{+} 3 \mu^{-}$. As we have already commented, there are no searches for this decay mode, and so neither constraints nor any direct way to estimate the potential of the $\mathrm{LHCb}$ to test this process. We therefore suggest the first analysis in this respect. 
TABLE I. Maximum and minimum efficiencies for selecting signal events in the channels $B_{s}^{0} \rightarrow 3 \mu^{+} 3 \mu^{-}\left(m_{X}=m_{B_{s}^{0}}\right)$ and $B^{+} \rightarrow K^{+} 3 \mu^{+} 3 \mu^{-}\left(m_{X}=m_{B^{+}}-m_{K^{+}}\right)$in each kinematic region. The upper limits $\left(\times 10^{-9}\right)$ on the corresponding branching ratios for $3 \mathrm{fb}^{-1}$ of data are also shown. We vary $m_{1,2}$ in the colored region of Fig. 2, with $m_{2}<10 \mathrm{GeV}$ and $m_{1} \geq 1.1 \mathrm{GeV}$. (For smaller values of $m_{1}$, the efficiency is negligible.)

\begin{tabular}{lccc}
\hline \hline & \multicolumn{2}{c}{$m_{X} \geq m_{1}+m_{2}$} & $m_{X}<m_{1}+m_{2}$ \\
\cline { 2 - 4 } & $m_{2} \geq 2 m_{1}$ & $m_{2}<2 m_{1}$ & $m_{X} \geq 3 m_{1}$ \\
\hline$B_{s}^{0} \rightarrow 3 \mu^{+} 3 \mu^{-}$ & {$[0.02,0.03]$} & {$[0.01,0.02]$} & {$[0.02,0.03]$} \\
Limit $\left(\times 10^{-9}\right)$ & {$[6.7,11.6]$} & {$[7.9,18.2]$} & {$[6.0,11.9]$} \\
$B^{+} \rightarrow K^{+} 3 \mu^{+} 3 \mu^{-}$ & {$[0.007,0.009]$} & {$[0.003,0.009]$} & Four-body \\
Limit $\left(\times 10^{-9}\right)$ & {$[5.9,8.0]$} & {$[6.0,16.6]$} & Four-body \\
\hline \hline
\end{tabular}

We first require events with at least one muon with $p_{T}>1.7 \mathrm{GeV}$; this cut ensures that the events pass the same hardware trigger used at $\sqrt{s}=8 \mathrm{TeV}$ [19]. We subsequently require exactly six muons, with vanishing total charge. We also require all muon tracks to have $p_{T}>0.5 \mathrm{GeV}$ and $2.5<\eta<5.0$. Finally, we require all muons tracks to have total momentum larger than $2.5 \mathrm{GeV}$ to simulate the threshold for muon identification based on the penetration power through absorption plates in the detector.

Due to the six muons in the final state, the SM backgrounds are negligible to very good approximation. They arise mostly from resonant production of $J / \Psi$ and $\varphi$ with subsequent decays into muons; we completely remove them by enforcing that no zero charge muon pair has an invariant mass in the range $[0.95,1.09] \cup$ $[3.0,3.2] \mathrm{GeV}$. (We lose sensitivity to signal events with $m_{1}$ in that region, though.) Even searches for four muons are background free $[19,21]$, so it is guaranteed that any observed event in the six lepton final state is due to the signal.

We generate signal $B$ meson events using PYTHIA v8 [25] and MadGraph v5 [26] with Feynrules v2 [27] for the decays. (We have cross-checked our event distributions using EvtGen [28].) Following Ref. [21], we compare the (mass dependent) efficiencies for selecting events in the channel $B_{s}^{0} \rightarrow 3 \mu^{+} 3 \mu^{-}$with that for $B_{s}^{0} \rightarrow 2 \mu^{+} 2 \mu^{-}$. The former is shown in Table I, while we estimate the latter to be $\varepsilon_{2 \mu^{+} 2 \mu^{-}} \sim 0.14$. The explanation for the smaller efficiencies for the six-muon process is twofold. First, due to the larger number of final state tracks, there are more events with no single muon with $p_{T}>1.7 \mathrm{GeV}$, which therefore do not pass the trigger; see Fig. 5. And second, there are more muons with at least one track with $p_{T}<0.5 \mathrm{GeV}$ which is therefore not detected; see Fig. 6.

Given the absence of background, we can estimate the upper limit on the branching ratio of the new processes at $\sqrt{s}=14 \mathrm{TeV}$ and luminosity $\mathcal{L}^{\prime}$ as

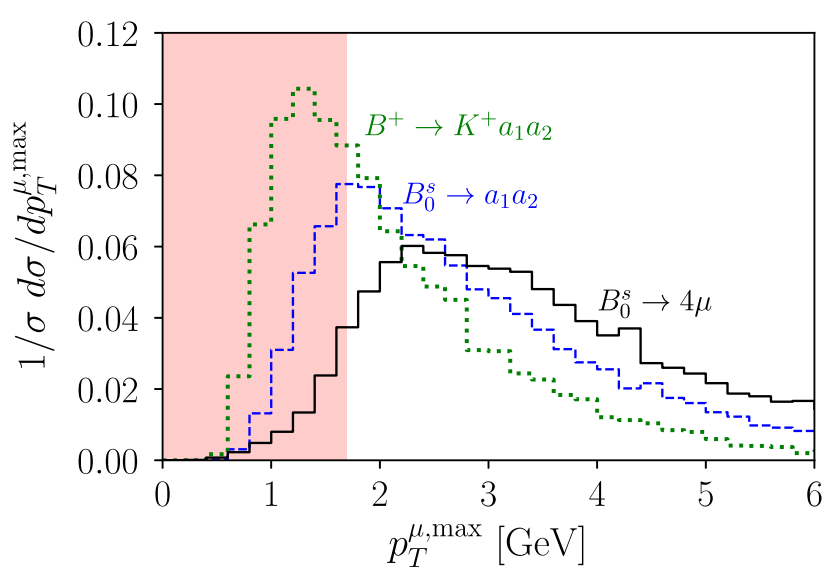

FIG. 5. Normalized distribution of the transverse momentum of the hardest muon for $B_{s}^{0} \rightarrow a_{1} a_{2}$ and $B^{+} \rightarrow K^{+} a_{1} a_{2}$ with $m_{1}=$ $1 \mathrm{GeV}$ and $m_{2}=2.5 \mathrm{GeV}$. These distributions are compared with the case $B_{0}^{s} \rightarrow 2 \mu^{+} 2 \mu^{-}$.

$$
\mathcal{B}_{\max }^{3 \mu^{+} 3 \mu^{-}} \sim \frac{\mathcal{B}_{\max }^{2 \mu^{+}+2 \mu^{-}} \times \varepsilon_{2 \mu^{+} 2 \mu^{-}}}{1.8 \times \varepsilon_{3 \mu^{+} 3 \mu^{-}}} \times \frac{\mathcal{L}}{\mathcal{L}^{\prime}},
$$

where $\mathcal{B}_{\max }^{2 \mu^{+} 2 \mu^{-}}$is the upper limit on $\mathcal{B}\left(B_{s}^{0} \rightarrow 2 \mu^{+} 2 \mu^{-}\right)=$ $2.5 \times 10^{-9}$, obtained in Ref. [19] with $\mathcal{L}=3 \mathrm{fb}^{-1}$ and $\sqrt{s}=8 \mathrm{TeV}$, under the same trigger and reconstruction criteria. The factor 1.8 stands for the approximated growth of the $b$ production cross section from $\sqrt{s}=8 \mathrm{TeV}$ to $\sqrt{s}=14 \mathrm{TeV}$. The prospective bounds on the branching ratio of this new decay mode are given in Table I.

We also consider the channel $B^{+} \rightarrow K^{+} 3 \mu^{+} 3 \mu^{-}$. In this case, on top of the selection criteria proposed before, we require the presence of a charged kaon which is also required to have $p_{T}>0.5 \mathrm{GeV}$ and $2.5<\eta<5.0$. The corresponding efficiencies are shown in Table I. The limit on the branching ratio can be again obtained as

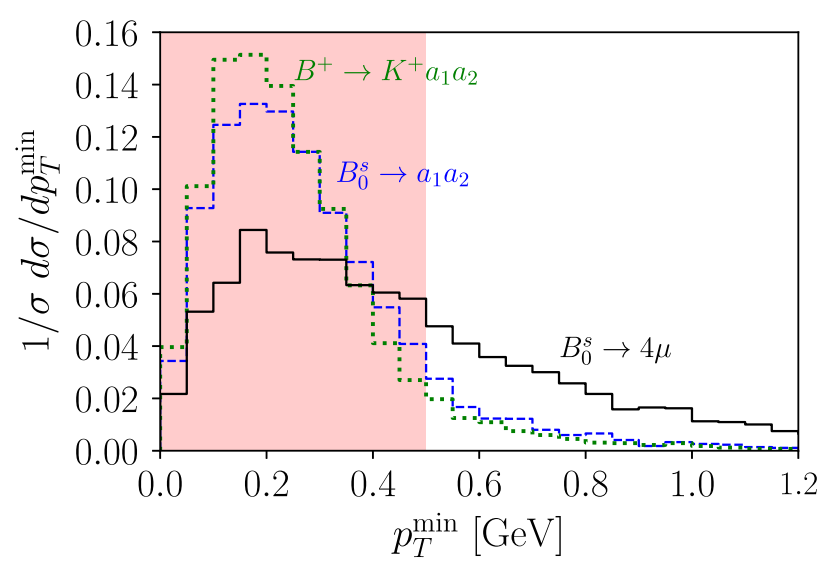

FIG. 6. Normalized distribution of the transverse momentum of the softest track for $B_{s}^{0} \rightarrow a_{1} a_{2}$ and $B^{+} \rightarrow K^{+} a_{1} a_{2}$ with $m_{1}=$ $1 \mathrm{GeV}$ and $m_{2}=2.5 \mathrm{GeV}$. These distributions are compared with the case $B_{0}^{s} \rightarrow 2 \mu^{+} 2 \mu^{-}$. 


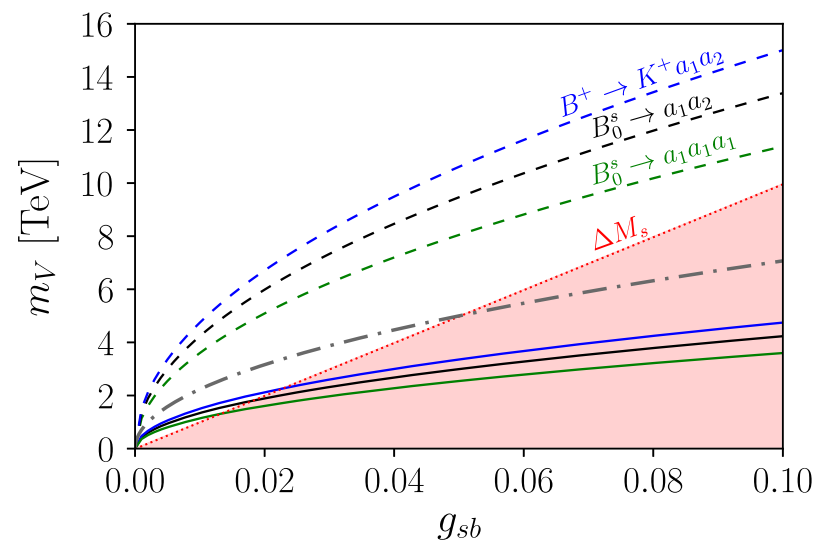

FIG. 7. Maximum value of $m_{V}$ that can be tested in the searches for $B_{s}^{0} \rightarrow 3 \mu^{+} 3 \mu^{-}$and $B^{+} \rightarrow K^{+} 3 \mu^{+} 3 \mu^{-}$at the current run of the $\mathrm{LHCb}$ (solid lines) and for Upgrade II (dashed lines). The red dotted line delimits the area excluded by measurements of $\Delta M_{s}$. In the dash-dotted line, the anomalies in $R_{K}$ and $R_{K^{*}}$ can be explained at the $1 \sigma$ level assuming $g_{V \ell \ell} \sim 1$ [30]. We have fixed $g_{12}=0.5$ as well as $m_{1}=1.2 \mathrm{GeV}$. We have set $m_{2}=2.0 \mathrm{GeV}$ for both $B_{0}^{s} \rightarrow a_{1} a_{2}$ and $B^{+} \rightarrow K^{+} a_{1} a_{2}$. For $B_{0}^{s} \rightarrow a_{1} a_{1} a_{1}$, we have fixed instead $m_{2}=m_{12}=5 \mathrm{GeV}$.

$$
\mathcal{B}_{\max }^{3 \mu^{+} 3 \mu^{-} K^{+}} \sim \frac{\mathcal{B}_{\max }^{2 \mu^{+}+2 \mu^{-}} \times \varepsilon_{2 \mu^{+} 2 \mu^{-}}}{1.8 \times 3.7 \times \varepsilon_{3 \mu^{+} 3 \mu^{-} K^{+}}} \times \frac{\mathcal{L}}{\mathcal{L}^{\prime}}
$$

where the factor 3.7 stands for the larger $B^{+}$production cross section [29]. The bounds obtained this way are also shown in Table I. It is worth noting that the prospective limits on this channel are comparable or even more stringent than that on the decay mode without the extra meson (due mostly to the larger cross section, that compensates the smaller efficiency). This fact, together with the observation that theoretically this decay mode dominates for $m_{2} \sim m_{1}$, strongly motivates searches for $B^{+} \rightarrow K^{+} 3 \mu^{+} 3 \mu^{-}$.

For illustration, we translate the expected limits in Table I to the plane $\left(g_{s b}, m_{V}\right)$ in Fig. 7 for definite values of $g_{12}, m_{1}, m_{2}$, and $m_{12}$ (when relevant). Prospects for the Upgrade II, defined by $\mathcal{L}^{\prime}=300 \mathrm{fb}^{-1}$, are also shown. It is interesting to see that with our proposed analyses we can easily test masses larger than $15 \mathrm{TeV}$, thereby outperforming constraints obtained from $\Delta M_{s}$ and completely probing the region in which the anomalies in lepton flavor universality can be explained.

Likewise, we also translate the aforementioned bounds to the plane $\left(m_{1}, m_{2}\right)$ in Fig. 8, fixing $g_{s b}=0.04$ as well as $m_{V}=4 \mathrm{TeV}$. Such values are not yet excluded by measurements of $\Delta M_{s}$; see Ref. [30]. In both figures, only the weakest limits of Table I are used.

We also note that, if a signal is observed in these sixmuon channels, the mass of the scalar particles involved could be reconstructed due to the outstanding detector resolution of the LHCb. To this aim, we provide two

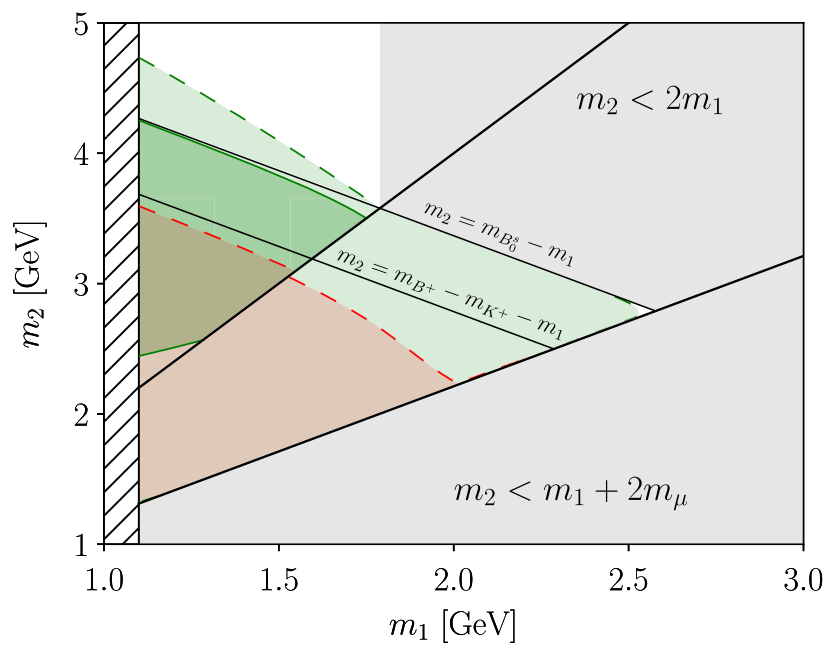

FIG. 8. Region of the plane $\left(m_{1}, m_{2}\right)$ that can be tested at the current run of the LHCb (solid) and in Upgrade II (dashed) in searches for $B_{s}^{0} \rightarrow 3 \mu^{+} 3 \mu^{-}$(green) and $B^{+} \rightarrow K^{+} 3 \mu^{+} 3 \mu^{-}$(red). We have fixed $g_{s b}=0.04, m_{V}=4 \mathrm{TeV}$, and $g_{12}=0.5$, as well as $m_{12}=1 \mathrm{GeV}$ (only relevant in the upper left region). The sensitivity is negligible in the slashed region.

different algorithms, depending on whether $m_{2}>2 m_{1}$ (in which case $a_{2} \rightarrow a_{1} a_{1}$ ) or rather $m_{2}<2 m_{1}$ (and therefore $a_{2} \rightarrow a_{1} \mu^{+} \mu^{-}$).

For the first case, we minimize the difference $\left|m_{11}^{\mathrm{rec}}-m_{12}^{\mathrm{rec}}\right|+\left|m_{12}^{\mathrm{rec}}-m_{13}^{\mathrm{rec}}\right|$, where $m_{i}^{\mathrm{rec}}$ is the invariant mass of each combination of opposite-sign muons. The two $a_{1} \mathrm{~s}$ that reconstruct the heavier scalar are those with the minimum $\Delta R$ among themselves; see Fig. 9 for an example.

Concerning the second case, the muon pairs reconstructing the two $a_{1} \mathrm{~s}$ are selected as those minimizing the difference $\left|m_{11}^{\text {rec }}-m_{12}^{\text {rec }}\right|$ among the three pairs of muons. Then, $a_{2}$ is reconstructed from the two muons not assigned to any $a_{1}$ and the $a_{1}$ that minimizes $\Delta R\left(p_{1}, p_{\mu \mu}\right)$ (with $p_{1}$

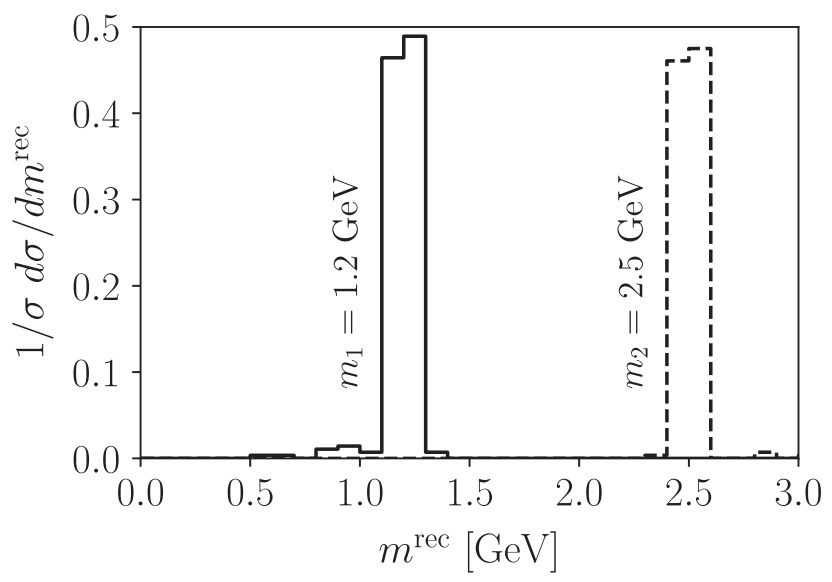

FIG. 9. Normalized distribution of the reconstructed $m_{1}$ (solid) and $m_{2}$ (dashed) for $m_{2}>2 m_{1}$. 


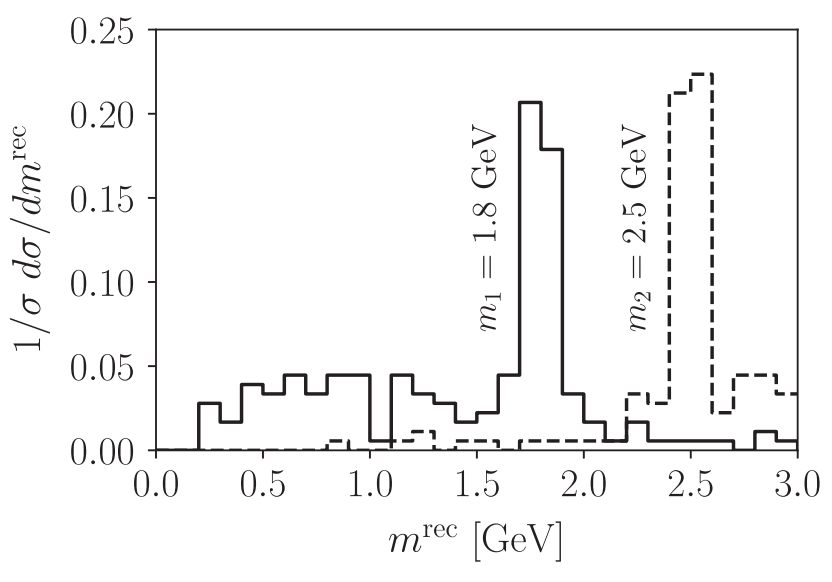

FIG. 10. Normalized distribution of the reconstructed $m_{1}$ (solid) and $m_{2}$ (dashed) for $m_{2}<2 m_{1}$.

its four momentum and $p_{\mu \mu}$ the four momentum of the aforementioned pair of muons); see Fig. 10.

\section{HIGH-MASS REGIME AT THE LHC}

In the high-mass regime, $a_{1,2}$ can no longer be produced in the decay of $B$ mesons. However, if $V$ is light enough $\left(m_{V} \lesssim\right.$ few $\left.\mathrm{TeV}\right)$, it can be produced on shell at colliders, giving rise to $a_{1,2}$ pair production upon decay. The tree level signal cross section for $g_{q q}=0.5$ and $g_{12}=1$ ranges between $\sim 0.04 \mathrm{pb}$ and $\sim 10^{-5} \mathrm{pb}$ for $m_{V}$ between 1 and $5 \mathrm{TeV}$.

There are multilepton searches at the LHC which are very sensitive to this scenario. Most of them rely on substantial missing energy, being therefore not relevant for our model. In this work, we consider the signal region dubbed SR0A in the analysis of Ref. [31]. The main selection cuts of that study are (i) at least four isolated leptons, (ii) no hadronic taus, (iii) no pair of opposite-sign leptons with invariant mass in the range $[81.2,101.2] \mathrm{GeV}$, and (iv) $m_{\text {eff }}>600 \mathrm{GeV}$, where $m_{\text {eff }}$ stands for the scalar sum of the $p_{T}$ of all leptons, jets with $p_{T}^{j}>40 \mathrm{GeV}$ and missing energy.

Only hadronic tau candidates with $p_{T}^{\tau}>20 \mathrm{GeV}$ are considered in (ii); jets are reconstructed using the anti- $k_{t}$ algorithm with $R=0.4$. The experimental analysis reports the observation of 13 events, while $10.2 \pm 2.1$ are predicted in the background-only hypothesis. Using these numbers including the systematic uncertainty on the SM prediction, we obtain that the maximum number of allowed signal events is 12. Scaling the expected number of background events with the larger luminosity, and assuming the same uncertainty, the expected maximum number of signal events at the HL-LHC is 300.

We recast this analysis using homemade routines based on ROOT v5 [32], HepMC v2 [33], and FasJet v3 [34]. We define hadronic taus as jets with angular separation smaller than 0.2 from a true hadronic decayed tau lepton.
We establish a flat tau-tagging efficiency of 0.5 . We consider light leptons to be isolated if the hadronic activity around $\Delta R=0.2$ of the corresponding lepton is smaller than $10 \%$ of its transverse momentum. On top of the cuts above, we require that the angular separation between any pair of muons is larger than 0.05 , to simulate their correct reconstruction at detectors.

We generate signal events for $p p \rightarrow V \rightarrow a_{1} a_{2}$ with the corresponding scalar decays with MadGraph v5 [26] with no parton level cuts. For the PDFs, we use the NNPDF23LO set [35]. Signal events are subsequently passed through PYTHIA v8 [25] to account for initial and final state radiation, fragmentation, and hadronization effects.

If the light scalars couple mostly to the tau lepton (second scenario introduced in Sec. II), the aforementioned signal region has no sensitivity. We can rely instead on the signal region SR2 defined in the same experimental paper of Ref. [31], which requires (i) exactly two light leptons with invariant mass not in the range $[81.2,101.2] \mathrm{GeV}$; (ii) at least two hadronic taus with $p_{T}^{\tau}>30 \mathrm{GeV}$; $m_{\text {eff }}>650 \mathrm{GeV}$. The experimental collaboration reports the observation of two events, the SM prediction being $2.3 \pm 0.8$. Using again the $\mathrm{CL}_{s}$ method, we obtain 6 (121) events as the current (future) maximum allowed signal.

We scan over 20 values of $m_{1}$ and $m_{2}$ in logarithmic scale in the range $[1,500] \mathrm{GeV}$, with special attention to low masses as well as masses close to the $Z$ pole.

In Fig. 11, we depict the region in the $\left(m_{1}, m_{2}\right)$ plane for $g_{q q}=0.5$ and $g_{12}=1$ that is already excluded in the muonphilic case and also in the case with couplings to taus. The exclusion prospects for the HL-LHC, defined by $3 \mathrm{ab}^{-1}$, are also shown. The tau analysis is much less constraining (mainly due to the small branching ratio to leptons), and thus we only show results for $m_{V}=1 \mathrm{TeV}$.

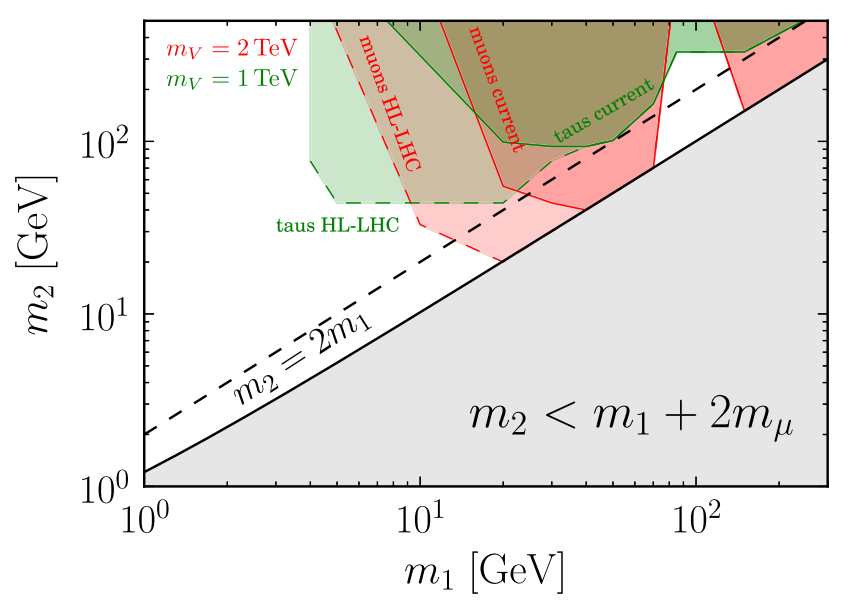

FIG. 11. Region in the plane $\left(m_{1}, m_{2}\right)$ that is excluded by multilepton searches (solid red) and lepton-tau searches (solid green) [31]. The dashed lines represent the corresponding prospects at the HL-LHC. We have fixed $g_{q q}=0.5, g_{12}=1$. 


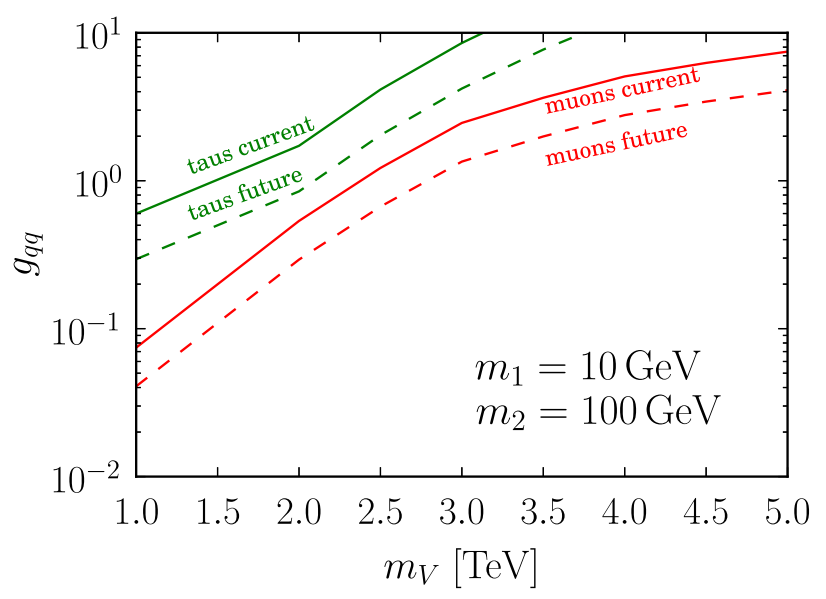

FIG. 12. Minimum value of $g_{q q}$ that it is excluded by multilepton searches (solid red) and lepton-tau searches (solid green) [31]. The dashed lines represent the corresponding prospects at the HL-LHC. We have assumed $\mathcal{B}\left(V \rightarrow a_{1} a_{2}\right) \sim 0.25$.

The low sensitivity in the small $m_{1}$ region is due to muons being very collimated. (Decays into taus are furthermore forbidden for $m_{1} \lesssim 4 \mathrm{GeV}$.) If it were possible to resolve muons with angular separations as small as 0.001 , then almost the whole small mass range could be tested in the muonphilic case.

Likewise, the nonexcluded region around $m_{1} \sim$ $100 \mathrm{GeV}$ results from the $Z$ veto of the analysis. This region could be covered if the veto on the $Z$ pole is removed and, instead of $m_{\mathrm{eff}}$, the invariant mass of all final state observable objects (which in our signal, and contrary to the SUSY targets of the analysis, presents a narrow peak) is used. Such improvement would also extend the reach to smaller masses. It is therefore desirable that future updates of the experimental work consider different versions of the cut on $m_{\text {eff }}$.

In the same vein, in Fig. 12, we plot the minimum value of $g_{q q}$ that can be tested for different values of $m_{V}$ and for fixed values of $m_{1,2}$. We have also fixed $g_{12}$ to the value for which $\mathcal{B}\left(V \rightarrow a_{1} a_{2}\right) \sim 0.25$.

\section{CONCLUSIONS}

We have studied the phenomenology of light leptophilic scalars $a_{1,2}$ that couple to a heavy flavor violating (mostly $b-s$ like) spin-1 resonance $V$. We have shown that, under very mild conditions, $a_{2}$ decays mostly into $a_{1}$, which subsequently decays into pairs of leptons. Thus, for scalar masses $\lesssim$ few $\mathrm{GeV}$, this scenario produces new $B$ meson decays into six muons, namely $B_{s}^{0} \rightarrow 3 \mu^{+} 3 \mu^{-}$and $B^{+} \rightarrow K^{+} 3 \mu^{+} 3 \mu^{-}$. Interestingly, the later dominates over the second when $m_{1} \sim m_{2}$. None of them has been explored experimentally; we have therefore proposed dedicated analyses to explore these signals at the LHCb. We have found that branching ratios as small as $6.0 \times 10^{-9}$ $\left(5.9 \times 10^{-9}\right)$ for the first (second) process can be already tested with the current luminosity. Branching ratios hundred times smaller could be probed at the Upgrade-II of the LHCb.

For larger scalar masses, $a_{1 / 2}$ arise rather in the decay of $V$, which can be produced on shell at $p p$ collisions at the LHC. Current multilepton searches in final states with muons (taus) constrain most of the parameter space for $m_{1} \gtrsim 10 \mathrm{GeV}$ provided that $\sigma\left(p p \rightarrow V \rightarrow a_{1} a_{2}\right) \gtrsim$ $0.001(0.01) \mathrm{pb}$. Smaller masses give rise to very collimated leptons (or jets) that are difficult to disentangle at detectors. However, at the HL-LHC, the reach can be extended to $m_{1} \lesssim 5$. And even further if the current analyses cut on the invariant mass of all visible objects.

Finally, let us comment how these results would get modified if different flavor assumptions are made. To start with, if $a_{1,2}$ are not leptophilic but rather they couple to all SM fermions with Yukawa-like couplings, the branching ratio of $a_{1}$ into leptons would get reduced by $1-2$ orders of magnitude. In turn, LHCb would be only sensitive to exotic branching ratios thousand times larger. (Note that such branching ratios are not excluded by any current measurement, though.) However, LHC searches in multilepton final states would lose almost all sensitivity in this case.

On the other hand, $V$ might also induce $b-d$ transitions. In that case, we expect new rare decays such as $B^{0} \rightarrow$ $3 \mu^{+} 3 \mu^{-}$. The production cross section for $B^{0}$ is $\sim 3.7$ larger than for $B_{s}^{0}$ [29], from where we estimate that $\mathcal{B}\left(B^{0} \rightarrow\right.$ $\left.3 \mu^{+} 3 \mu^{-}\right) \gtrsim 1.6 \times 10^{-9}\left(\sim 10^{-11}\right)$ can be probed currently (in the Upgrade-II of the LHCb).

On the theory side, this channel vanishes also at tree level when $m_{1} \sim m_{2}$. In this regime, we propose searching for $B_{s}^{0} \rightarrow K^{* 0} 3 \mu^{+} 3 \mu^{-}$, with $K^{* 0} \rightarrow K^{+} \pi^{-}$, whose branching ratio is around $2 / 3$ [36]. Upon performing an equivalent analysis to that described in Sec. III, we obtain efficiencies of about 2 times smaller, in comparison to the $B_{s}^{0} \rightarrow$ $3 \mu^{+} 3 \mu^{-}$channel. Consequently, we estimate the $\mathrm{LHCb}$ reach to be $\mathcal{B}\left(B_{s}^{0} \rightarrow K^{* 0} 3 \mu^{+} 3 \mu^{-}\right) \gtrsim 1.8 \times 10^{-8}$ currently and again about hundred times stronger in the Upgrade-II.

At high scalar masses, the prospects are only slightly better than for $b-s$ transitions, because the production cross section for $V$ at the LHC grows only by a very small factor. Both low and high energy searches are also more constraining than bounds on $\Delta M_{d}$ [37] on a wide region of the parameter space.

Overall, our study motivates new searches for $B_{s}^{0} \rightarrow$ $\left(K^{0 *}\right) 3 \mu^{+} 3 \mu^{-}$and $B^{+} \rightarrow K^{+} 3 \mu^{+} 3 \mu^{-}$at the LHCb as well as small modifications of current multilepton and multitau analyses at CMS and ATLAS.

\section{ACKNOWLEDGMENTS}

We are grateful to Ulrik Egede for previous collaboration that opened this new line of research. M. C. is supported by the Royal Society under the Newton International Fellowship programme. M. R. is supported by Fundação 
para a Ciência e Tecnologia (FCT) under the Grant No. PD/ $\mathrm{BD} / 142773 / 2018$ and also acknowledges financing from LIP (FCT, COMPETE2020-Portugal2020, FEDER, POCI01-0145-FEDER-007334). M. R. would like to thank the IPPP Durham for hospitality where the main part of this work was carried out. M. S. acknowledges the hospitality of the University of Tuebingen and support of the Humboldt Society during the completion of parts of this work.

\section{APPENDIX: CONCRETE COMPOSITE HIGGS MODEL}

Nonminimal CHMs is the context where heavy vector bosons and new light scalars, separated by a large mass gap, arise more naturally. The reason is that the latter are pseudo-Nambu-Golstone bosons (pNGBs) from the spontaneous breaking of $\mathcal{G} / \mathcal{H}$, at a scale $f \sim \mathrm{TeV}$.

The smallest coset for which the scalar sector consists of the Higgs degrees of freedom as well as two SM singlets is $S O(7) / S O(6)$ [7-9]. The corresponding 15 unbroken and 6 broken generators, $T$ and $X$, respectively, can be written as

$T_{i j}^{m n}=-\frac{i}{\sqrt{2}}\left(\delta_{i}^{m} \delta_{j}^{n}-\delta_{i}^{n} \delta_{j}^{m}\right), \quad m<n \in[1,6]$,

$X_{i j}^{m n}=-\frac{i}{\sqrt{2}}\left(\delta_{i}^{m} \delta_{j}^{7}-\delta_{i}^{7} \delta_{j}^{m}\right), \quad m \in[1,6]$.

Without loss of generality, the pNGB matrix can be written as

$$
U=\left[\begin{array}{ccccc}
\mathbf{1}_{3 \times 3} & & & & \\
& 1-h^{2} /\left(f^{2}+f^{2} \Sigma\right) & -h a_{1} /\left(f^{2}+f^{2} \Sigma\right) & -h a_{2} /\left(f^{2}+f^{2} \Sigma\right) & h / f \\
-h a_{1} /\left(f^{2}+f^{2} \Sigma\right) & 1-a_{1}^{2} /\left(f^{2}+f^{2} \Sigma\right) & -a_{1} a_{2} /\left(f^{2}+f^{2} \Sigma\right) & a_{1} / f \\
-h a_{2} /\left(f^{2}+f^{2} \Sigma\right) & -a_{1} a_{2} /\left(f^{2}+f^{2} \Sigma\right) & 1-a_{2}^{2} /\left(f^{2}+f^{2} \Sigma\right) & a_{2} / f \\
-h / f & -a_{1} / f & -a_{2} / f & \Sigma
\end{array}\right]
$$

with $\Sigma^{2}=1-\left(h^{2}+a_{1}^{2}+a_{2}^{2}\right) / f^{2}$.

Following the partial compositeness paradigm [38], the couplings of $a_{1,2}$ to the SM fermions, as well as the scalar potential, depend on the quantum numbers of the composite operators that the SM fermions mix with breaking the global symmetry. Or equivalently, they depend on how the SM fermions are embedded in representations of $\mathrm{SO}(7)$.

We assume that $q_{L}+u_{R} \sim 7+\mathbf{2 1}$. Likewise, we assume that $l_{L}+e_{R} \sim 27+1$. Explicitly, $L_{L} \equiv \nu_{L} \Lambda^{e}+e_{L} \Lambda^{\nu}$,

$$
L_{L}=\frac{1}{2}\left(\begin{array}{cccc}
0_{4 \times 4} & \theta \mathbf{v}_{1}{ }^{T} & \gamma \mathbf{v}_{2}{ }^{T} & \mathbf{v}_{2}{ }^{T} \\
\theta \mathbf{v}_{1} & 0 & 0 & 0 \\
\gamma \mathbf{v}_{2} & 0 & 0 & 0 \\
\mathbf{v}_{2}{ }^{T} & 0 & 0 & 0
\end{array}\right),
$$

where the vectors read $\mathbf{v}_{1}=\left(e_{L},-i e_{L}, \nu_{L}, i \nu_{L}\right)$ and $\mathbf{v}_{2}=$ $\left(i e_{L}, e_{L}, i \nu_{L},-\nu_{L}\right)$ and $\theta$ and $\gamma$ are real parameters. (Note that the different embeddings for quarks and leptons are primarily justified by the fact that the lepton and quark masses and mixings are completely different.)

The scalar potential can be written as $V\left(h, a_{1,2}\right)=$ $V_{q}\left(h, a_{1,2}\right)+V_{l}\left(h, a_{1,2}\right)$, where the first and second contributions of the rhs come from loops of quarks and leptons, respectively. It can be also shown that the quark sector respects a symmetry $a_{1,2} \rightarrow-a_{1 / 2}$, as well as the shift symmetry of the singlets. Consequently, $V_{q}\left(h, a_{1,2}\right)=V_{q}(h)$. It is completely fixed by the measurements of the Higgs mass and its vacuum expectation value.
The only model dependence come from $V_{l}\left(h, a_{1,2}\right)$, which to leading order in the expansion in the global symmetry breaking parameters reads

$$
V_{l} \sim c_{1} f^{4}\left[\left(\Lambda_{D}^{\mathbf{1} *}\right)^{\alpha}\left(\Lambda_{D}^{\mathbf{1}}\right)_{\alpha}\right]+c_{2} f^{4}\left[\left(\Lambda_{D}^{\mathbf{6} *}\right)_{i}^{\alpha}\left(\Lambda_{D}^{\mathbf{6}}\right)_{\alpha}^{i}\right],
$$

where the dressed spurion reads $\Lambda_{D}^{\alpha} \equiv U^{T} \Lambda^{\alpha} U$ with $\alpha=e$, $\nu$. (The indices 1 and 6 indicate the projection into the singlet and the sextuplet in the decomposition $\mathbf{2 7}=1+$ $\mathbf{6}+\mathbf{2 0}$ from $S O(7)$ to $S O(6)$.) The constants $c_{1}$ and $c_{2}$ are free parameters encoding the (unknown) details on the strongly coupled UV. Writing explicitly the one-loop induced potential, we find

$$
\begin{aligned}
V_{l}= & 4 f^{3} c_{2} \gamma \Sigma a_{2}+2 f\left(c_{1}-2 c_{2}\right) \gamma \Sigma a_{2} h^{2} \\
& +\frac{1}{2} c_{2} f^{2}\left[\left(\gamma^{2}+\theta^{2}-7+2 \frac{c_{1}}{c_{2}}\right) h^{2}\right. \\
& \left.+4\left(\theta^{2}-1\right) a_{1}^{2}+4\left(\gamma^{2}-1\right) a_{2}^{2}\right] \\
& +\left(c_{1}-2 c_{2}\right)\left[\left(\theta^{2}-1\right) a_{1}^{2}+\left(\gamma^{2}-1\right) a_{2}^{2}-h^{2}\right] h^{2} .
\end{aligned}
$$

We further expand this expression in powers of $1 / f$, and keep only terms up to dimension four,

$$
\begin{aligned}
V_{l} \sim & 4 f^{3} c_{2} \gamma a_{2}+2 f^{2} c_{2}\left[\left(\gamma^{2}-1\right) a_{2}^{2}+\left(\theta^{2}-1\right) a_{1}^{2}\right] \\
& +2 f \gamma\left[\left(c_{1}-3 c_{2}\right) a_{2} h^{2}-c_{2}\left(a_{1}^{2}+a_{2}^{2}\right) a_{2}\right] \\
& +\left(c_{1}-2 c_{2}\right)\left[\left(\theta^{2}-1\right) a_{1}^{2}+\left(\gamma^{2}-1\right) a_{2}^{2}\right] h^{2}+\ldots
\end{aligned}
$$




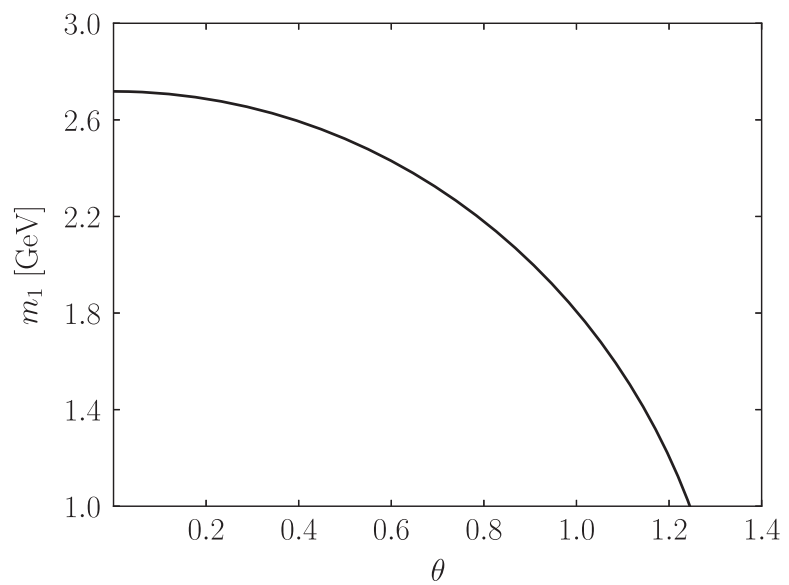

FIG. 13. Mass of $a_{1}$ as a function of $\theta$ (for $f=1 \mathrm{TeV}$ ), obtained from the embedding of the left-handed leptons in the symmetric representation of $S O(7)$.

where the three dots encode terms involving the Higgs boson solely.

The requirements $c_{1} \sim 3 c_{2}$ and $\gamma \sim 1$ make the interactions between $a_{2}$ and the Higgs (in particular mixings) very small. In order to avoid bounds from Higgs searches, we restrict to this case hereafter. The tadpole can then be removed by the field redefinition $a_{2} \rightarrow a_{2}+\sqrt{2 / 3} f$.

Let us also fix $f=1 \mathrm{TeV}$, as well as $c_{2} \sim g_{*}^{2} y_{l}^{2} /$ $(4 \pi)^{2} \sim 10^{-6}$. The latter is the value expected from strongly-interacting light Higgs power counting [39] for $g_{*} \sim 3$, with $g_{*}$ the typical strong coupling between composite resonances. This choice fixes both $m_{2}$ and $m_{12}$ to $\sim 3.1$ and $\sim 0.002 \mathrm{GeV}$, respectively; while $m_{1}$ depends solely on $\theta$. We compute numerically this dependence and it is depicted in Fig. 13.

On another front, the Yukawa Lagrangian to dimension five reads

$$
\begin{aligned}
L_{Y} & =y f{\overline{l_{L}}}^{\alpha}\left(\Lambda_{D}^{\alpha}\right)_{77}^{\dagger} e_{R}+\text { H.c. } \\
& =y_{\ell} \overline{l_{L}} H e_{R}\left[1+\frac{1}{f}\left(a_{2}-i \theta a_{1}\right)+\ldots\right] .
\end{aligned}
$$

The vector resonance associated to the generator $T^{56}$ is the only one that couples to $a_{1,2}$. We identify it with $V$. The interaction between $V$ and the pNGBs is entirely determined by the Callan-Coleman-Wess-Zumino formalism [40-42], reading

$$
L=\frac{1}{2 g_{*}^{2}} m_{V}^{2}\left(g_{*} V_{\mu}^{a}-e_{\mu}^{a}\right)^{2}
$$

where $e_{\mu}$ is the trace of the Maurer-Cartan form $\omega$ along the unbroken generators

$$
\omega_{\mu}=-i U^{\dagger} \partial_{\mu} U=d_{\mu}^{a} X_{a}+e_{\mu}^{a} T_{a} .
$$

We expect $m_{V} \sim g_{*} f$. Therefore, the interaction between the vector resonance and the light scalars reads

$$
L_{\text {int }}=\sqrt{2} g_{*} \frac{V_{\mu} a_{2} \overleftrightarrow{\partial^{\mu}} a_{1}}{1+\Sigma} \sim \frac{g_{*}}{\sqrt{2}} V_{\mu} a_{2} \overleftrightarrow{\partial^{\mu}} a_{1}
$$

Finally, the vector resonance cannot couple directly to the left-handed quarks. The coupling $g_{q q}$ is therefore suppressed by $v^{2} / f^{2} \lesssim 0.1$.

Altogether, this model matches into the parametrization in Eq. (1). For example, let us take $\theta=1.2$. We obtain $m_{1} \sim 1.3 \mathrm{GeV}, m_{2} \sim 3.1 \mathrm{GeV}, m_{12} \sim 0.002 \mathrm{GeV}, g_{q q} \sim 0.1$, $g_{12} \sim 2, g_{2} \sim 0.17, g_{1} \sim 0.22$.

These numbers are also obtained if the leptons are embedded in $\mathbf{7}+\mathbf{7}$.
[1] M. Chala, A critical assessment of the status of LHC searches for new physics, Proc. Sci., CORFU2017 (2018) 047.

[2] D. B. Kaplan and H. Georgi, SU(2) $\times U(1)$ Breaking by vacuum misalignment, Phys. Lett. 136B, 183 (1984).

[3] D. B. Kaplan, H. Georgi, and S. Dimopoulos, Composite Higgs scalars, Phys. Lett. 136B, 187 (1984).

[4] B. Gripaios, A. Pomarol, F. Riva, and J. Serra, Beyond the minimal composite Higgs model, J. High Energy Phys. 04 (2009) 070.

[5] L. Vecchi, The natural composite Higgs, arXiv:1304.4579.

[6] V. Sanz and J. Setford, Composite Higgses with seesaw EWSB, J. High Energy Phys. 12 (2015) 154.
[7] M. Chala, G. Nardini, and I. Sobolev, Unified explanation for dark matter and electroweak baryogenesis with direct detection and gravitational wave signatures, Phys. Rev. D 94, 055006 (2016).

[8] R. Balkin, M. Ruhdorfer, E. Salvioni, and A. Weiler, Charged composite scalar dark matter, J. High Energy Phys. 11 (2017) 094.

[9] L. Da Rold and A. N. Rossia, The minimal simple composite Higgs model, arXiv:1904.02560.

[10] C. Niehoff, P. Stangl, and D. M. Straub, Violation of lepton flavour universality in composite Higgs models, Phys. Lett. B 747, 182 (2015). 
[11] C. Niehoff, P. Stangl, and D. M. Straub, Direct and indirect signals of natural composite Higgs models, J. High Energy Phys. 01 (2016) 119.

[12] A. Carmona and F. Goertz, Lepton Flavor and Nonuniversality from Minimal Composite Higgs Setups, Phys. Rev. Lett. 116, 251801 (2016).

[13] E. Megias, G. Panico, O. Pujolas, and M. Quiros, A natural origin for the LHCb anomalies, J. High Energy Phys. 09 (2016) 118.

[14] I. Garcia Garcia, LHCb anomalies from a natural perspective, J. High Energy Phys. 03 (2017) 040.

[15] F. Sannino, P. Stangl, D. M. Straub, and A. E. Thomsen, Flavor physics and flavor anomalies in minimal fundamental partial compositeness, Phys. Rev. D 97, 115046 (2018).

[16] A. Carmona and F. Goertz, Recent $B$ physics anomalies: A first hint for compositeness? Eur. Phys. J. C 78, 979 (2018).

[17] M. Chala and M. Spannowsky, Behavior of composite resonances breaking lepton flavor universality, Phys. Rev. D 98, 035010 (2018).

[18] S. V. Demidov and D. S. Gorbunov, Flavor violating processes with sgoldstino pair production, Phys. Rev. D 85, 077701 (2012).

[19] R. Aaij et al. (LHCb Collaboration), Search for decays of neutral beauty mesons into four muons, J. High Energy Phys. 03 (2017) 001.

[20] A. E. Nelson and J. Scholtz, Heavy flavor and dark sector, Phys. Rev. D 91, 014009 (2015).

[21] M. Chala, U. Egede, and M. Spannowsky, Searching new physics in rare $B$-meson decays into multiple muons, Eur. Phys. J. C 79, 431 (2019).

[22] J. Liu, C. E. M. Wagner, and X.-P. Wang, A light complex scalar for the electron and muon anomalous magnetic moments, J. High Energy Phys. 03 (2019) 008.

[23] K. Cheung, C.-W. Chiang, N. G. Deshpande, and J. Jiang, Constraints on flavor-changing Z' models by B(s) mixing, Z' production, and $\mathrm{B}(\mathrm{s})->\mathrm{mu}+\mathrm{mu}-$, Phys. Lett. B 652, 285 (2007).

[24] P. Ball and R. Zwicky, New results on $B \rightarrow \pi, K, \eta$ decay formfactors from light-cone sum rules, Phys. Rev. D 71, 014015 (2005).

[25] T. Sjöstrand, S. Ask, J. R. Christiansen, R. Corke, N. Desai, P. Ilten, S. Mrenna, S.Prestel, C. O. Rasmussen, and P.Z. Skands, An introduction to PYTHIA 8.2, Comput. Phys. Commun. 191, 159 (2015).

[26] J. Alwall, R. Frederix, S. Frixione, V. Hirschi, F. Maltoni, O. Mattelaer, H.-S. Shao, T. Stelzer, P. Torrielli, and M. Zaro, The automated computation of tree-level and next-toleading order differential cross sections, and their matching to parton shower simulations, J. High Energy Phys. 07 (2014) 079.

[27] C. Degrande, C. Duhr, B. Fuks, D. Grellscheid, O. Mattelaer, and T. Reiter, UFO-The Universal FeynRules output, Comput. Phys. Commun. 183, 1201 (2012).

[28] D. J. Lange, The EvtGen particle decay simulation package, Nucl. Instrum. Methods Phys. Res., Sect. A 462, 152 (2001).

[29] R. Aaij et al. (LHCb Collaboration), Measurement of $b$-hadron production fractions in $7 \mathrm{TeV}$ pp collisions, Phys. Rev. D 85, 032008 (2012).

[30] L. Di Luzio, M. Kirk, and A. Lenz, Updated $B_{s}$-mixing constraints on new physics models for $b \rightarrow s \ell^{+} \ell^{-}$anomalies, Phys. Rev. D 97, 095035 (2018).

[31] M. Aaboud et al. (ATLAS Collaboration), Search for supersymmetry in events with four or more leptons in $\sqrt{s}=$ $13 \mathrm{TeV} p p$ collisions with ATLAS, Phys. Rev. D 98, 032009 (2018).

[32] R. Brun and F. Rademakers, ROOT: An object oriented data analysis framework, Nucl. Instrum. Methods Phys. Res., Sect. A 389, 81 (1997).

[33] M. Dobbs and J. B. Hansen, The HepMC C++ Monte Carlo event record for high energy physics, Comput. Phys. Commun. 134, 41 (2001).

[34] M. Cacciari, G. P. Salam, and G. Soyez, FastJet user manual, Eur. Phys. J. C 72, 1896 (2012).

[35] R. D. Ball et al., Parton distributions with LHC data, Nucl. Phys. B867, 244 (2013).

[36] M. Tanabashi et al. (Particle Data Group), Review of particle physics, Phys. Rev. D 98, 030001 (2018).

[37] P. Foldenauer and J. Jaeckel, Purely flavor-changing Z' bosons and where they might hide, J. High Energy Phys. 05 (2017) 010.

[38] D. B. Kaplan, Flavor at SSC energies: A new mechanism for dynamically generated fermion masses, Nucl. Phys. B365, 259 (1991).

[39] G. F. Giudice, C. Grojean, A. Pomarol, and R. Rattazzi, The strongly-interacting light Higgs, J. High Energy Phys. 06 (2007) 045.

[40] S. R. Coleman, J. Wess, and B. Zumino, Structure of phenomenological Lagrangians. 1, Phys. Rev. 177, 2239 (1969).

[41] C. G. Callan, Jr., S. R. Coleman, J. Wess, and B. Zumino, Structure of phenomenological Lagrangians. 2, Phys. Rev. 177, 2247 (1969).

[42] G. Panico and A. Wulzer, The composite Nambu-Goldstone Higgs, Lect. Notes Phys. 913, 1 (2016). 